\title{
Correction to: Mechano-Immunomodulation: Mechanoresponsive Changes in Macrophage Activity and Polarization
}

\author{
Sarah Adams, ${ }^{1}$ Leah M. Wuescher, ${ }^{2}$ Randall Worth, ${ }^{2}$ and Eda Yildirim-Ayan ${ }^{1,3}$ \\ ${ }^{1}$ Department of Bioengineering, College of Engineering, University of Toledo, Toledo, OH 43606, USA; ${ }^{2}$ Department of \\ Medical Microbiology and Immunology, University of Toledo College of Medicine and Life Sciences, Toledo, OH 43614, USA; \\ and ${ }^{3}$ Department of Orthopaedic Surgery, University of Toledo Medical Center, Toledo, OH 43614, USA
}

\section{Correction to: Annals of Biomedical Engineering (2019)} https://doi.org/10.1007/s10439-019-02302-4

This article was corrected to add a permissions credit to Figure 3 legend.
Publisher's Note Springer Nature remains neutral with regard to jurisdictional claims in published maps and institutional affiliations.

Address correspondence to Eda Yildirim-Ayan, Department of Bioengineering, College of Engineering, University of Toledo, Toledo, OH 43606, USA. Electronic mail: eda.yildirimayan@utoledo.edu

The original article can be found online at https://doi.org/10. 1007/s10439-019-02302-4. 